\title{
Review \\ Progesterone receptors - animal models and cell signalling in breast cancer Diverse activation pathways for the progesterone receptor: possible implications for breast biology and cancer
}

\author{
Claudia Lanari and Alfredo A Molinolo
}

Laboratory of Hormonal Carcinogenesis, Instituto de Biología y Medicina Experimental, Consejo Nacional de Investigaciones Cientificas y Técnicas, Buenos Aires, Argentina

Correspondence: Alfredo A Molinolo, Instituto de Biología y Medicina Experimental, Vuelta de Obligado 2490, C1428ADN Buenos Aires, Argentina. Tel: +54 114783 2869; fax: +54 114786 2564; e-mail: molinolo@dna.uba.ar

Received: 18 April 2002

Breast Cancer Res 2002, 4:240-243 (DOI 10.1186/bcr539)

Revisions requested: 13 May 2002

Revisions received: 16 August 2002

Accepted: 21 August 2002

(C) 2002 BioMed Central Ltd

Published: 12 September 2002

(Print ISSN 1465-5411; Online ISSN 1465-542X)

\begin{abstract}
Progesterone and estradiol, and their nuclear receptors, play essential roles in the physiology of the reproductive tract, the mammary gland and the nervous system. Estrogens have traditionally been considered associated with an increased risk of breast cancer. There is, however, compelling evidence that progesterone plays an important role in breast cell proliferation and cancer. Herein, we review the possible role of progestins and the progesterone receptor-associated signaling pathways in the development of breast cancer, as well as the therapeutic possibilities arising from our growing knowledge of the activation of the progesterone receptor by other proliferative mechanisms.
\end{abstract}

Keywords: antiprogestins, breast cancer, crosstalk, ligand independent, medroxyprogesterone, progesterone receptor

\section{Introduction}

Progesterone and estradiol, and their cognate receptors, play essential roles in systems as diverse as the reproductive tract, the mammary gland and the nervous system. The best understood role of ovarian steroid hormones is probably their participation in reproductive tract physiology. Estrogens drive the proliferation of the endometrium after menses, and induce progesterone receptor (PR) expression, whereas progesterone plays a role in proliferation, differentiation and maintenance of the endometrial epithelium and stroma in preparation for implantation.

The classic view (namely, estrogens $=$ proliferation and progestins $=$ differentiation) has been extrapolated to systems other than the endometrium, such as the mammary gland, and has probably contributed to a longheld belief that estrogens are the main steroid hormones implicated in the induction of breast cancer. However, this concept has been challenged by growing experimental evidence in rodents. This evidence points towards progesterone and its related signaling pathways as important players in the induction, progression and maintenance of the neoplastic phenotype in the mammary gland [1]. Moreover, recently available clinical data demonstrate a higher risk of breast cancer in patients under hormone replacement therapy using a combination of estrogens and progestins, as compared with those using estrogens alone $[2,3]$. The PR has emerged as a major player in the field of breast cancer.

As with the estrogen receptor (ER) signaling pathway, blocking the progesterone/PR system, alone or in combination with other treatment modalities, has arisen as a logical therapeutic possibility in breast cancer [4]. 


\section{PR signaling in breast cancer development: experimental support In vivo studies}

We demonstrated in 1986 [5] that, in BALB/c female mice, medroxyprogesterone acetate (MPA) alone induces mammary carcinomas that expresses PRs and ERs, that are hormone dependent and that, unlike what happens in most other experimental models of breast cancer, originate axillary and lung metastases [6,7]. This was the first, and as far as we know the only, time that a progestin alone was demonstrated to induce hormone-dependent mammary cancer. No tumors were observed in untreated controls or in virgin female BALB/c mice of our colony. Some of these tumors have been maintained by subcutaneous passages and they still maintain the steroid receptor expression.

At about the same time, Nagasawa et al. [8] showed that MPA enhanced spontaneous mammary tumorigenesis in SHN mice, but they did not report on steroid receptor expression or hormone sensitivity. In 1993, we were able to induce mammary carcinomas in BALB/c female mice using progesterone [9]. Most of the tumors were lobular in morphology and they progressively ceased to express receptors after a few in vivo passages. In mice, progestins can act as co-carcinogens together with chemical initiators, such as $\mathrm{N}$-methyl- $\mathrm{N}$-nitrosourea [10] and dimethylbenzanthracene [11]. Recent studies in PR knockout mice confirmed the role of PR on dimethylbenzanthraceneinduced tumorigenesis [12]. There is evidence in rats that, depending on the time of carcinogen administration in relation to hormone treatment, progesterone can act as a promoter [13].

\section{In vitro studies}

Progestins have been demonstrated to exert variable effects on different cell types depending on environmental factors and culture conditions. Progesterone increases DNA synthesis in mouse mammary gland organ culture, decreases proliferation in primary cultures of normal human breast epithelium and cultured breast cancer cells, increases cell proliferation under certain experimental conditions in T47-D cells [14] and increases cell proliferation in primary cultures of experimental mouse mammary tumors [15].

\section{Dissecting the PR signaling pathway Progesterone action in the target cell}

The physiological effects of progesterone are mediated by specific intracellular proteins known as PRs. In rodents and humans, the PR gene encodes two proteins termed $\mathrm{PR}_{\mathrm{A}}$ and $\mathrm{PR}_{\mathrm{B}}$. Both isoforms are the result of transcription of two alternative promoters and initiation of translation at two different AUG codons. Their physiological roles are different according to their structural and functional properties. $\mathrm{PR}_{\mathrm{A}}$ and $\mathrm{PR}_{\mathrm{B}}$ may activate different genes, and their ratio of expression may be important in cell fate $[16,17]$.
Activated PRs would recruit a series of important regulatory proteins, which can serve as coactivators or corepressors, such as SRC-1, SRC-2 and SRC-3, CBP/p300 and others. These coregulatory proteins may modulate histone acetylation/deacetylation and chromatin remodeling, and may have additional effects [18]. The PR complex will bind a specific DNA sequence, the progesterone-responsive element, and will initiate the transcription of target genes. A complete review of the classical mechanism by which PRs are activated by their natural ligands is beyond the scope of the present review, and has been extensively described elsewhere [19]. Such a complex activation sequence offers several steps in which other regulatory mechanisms of the progesterone signaling pathway can be integrated.

\section{PR activation}

Four sites of the PR are basally phosphorylated in humans (Ser 81, Ser 162, Ser 190 and Ser 400), and exhibit a rapid twofold increase on hormone treatment. The other sites (Ser 102, Ser 294 and Ser 345) are hormone inducible, and 1-2 hours of treatment are required to reach maximal phosphorylation. Their different kinetics in response to hormone suggests that these two groups of phosphorylation sites are targets of different signaling pathways and kinases, and serve distinct functional structural roles. Phosphorylation may not serve as a regulatory on-off switch for transcriptional activity, but rather functions to either amplify or attenuate activity [20].

Several crosstalk mechanisms involving the conventional nuclear PR pathway with different growth factors, neurotransmitters and polypeptide hormones have been described. Most studies indicate that progestins upregulate growth factor and cytokine receptors at the cell surface. They also act at the cytoplasmic level to regulate several intracellular effectors by increasing the levels and altering the subcellular compartmentalization of, for example, Stat 5 , and by potentiating mitogen-activated protein kinase (MAPK) and Janus kinase activities [14]. In addition, at the nuclear level, growth factor-regulated nuclear transcription factors may synergize with agonistoccupied PRs to modulate the activity of key genes involved in breast cancer fate [14]. All these studies deal with increases in sensitivity to different proliferative signals, rather than a ligand-independent interaction with PRs. Evidence of a direct PR activation by the protein kinase A pathway was provided by Edwards et al. [21] in the T-47D cell line. Recently, Jacobsen et al. [22] studied ligand-independent activation of the $\mathrm{PR}_{\mathrm{A}}$, which resulted in increased expression of several genes, among them prolactin receptors.

In a nonengineered system, we have provided evidence of crosstalk between the PR and basic fibroblast growth factor using primary cultures of MPA-induced mammary 
carcinomas. The stimulatory effect of MPA could be mimicked by basic fibroblast growth factor [15], and these effects could be blocked by antiprogestins or by PR antisense oligonucleotides. This indicates that fibroblast growth factors, which act by activation of MAPK, may also use the PR pathway to induce cell proliferation. These results are also supported by data from Elizalde et al. [23], who recently demonstrated that heregulin, which also activates MAPK, is able to induce PR mobility gel shift or to activate luciferase in C4-HD cells, a MPA-responsive tumor line transfected with a progesterone-responsive element luciferase reporter gene.

Steroid receptors may crosstalk with each other. Migliaccio et al. [24] described an interaction between $\mathrm{PR}_{\mathrm{B}}$, $\mathrm{ER} \alpha$ and Src at the cell membrane level, which would be necessary for steroid-induced S-phase entry of cells. PR nongenomic mechanisms of action have been described in different systems [25], but not yet in the mammary gland.

All studies regarding crosstalks and PRs in the mammary gland were performed in vitro. In vivo demonstration of these mechanisms will strengthen the role of the PR on mammary gland or breast cancer proliferation.

\section{Antiprogestins: experimental and clinical studies}

Mifepristone demonstrated dose-dependent growth inhibitory effects in vitro in PR-positive human breast cancer cell lines, and its antiproliferative effects were evident even in the total absence of estrogens [26]. We were able to demonstrate complete regression of experimental MPA-induced metastatic tumors with antiprogestins in vivo [27]. Additive antitumor effects using onapristone in combination with tamoxifen or the pure antiestrogen ICl 164384 have been demonstrated in several experimental models [28].

In clinical studies, from 169 patients treated, a complete response was observed in only one patient, and partial response rates varied between 11 and 56\% [4]. Interestingly, patients resistant to treatment with tamoxifen or high-dose progestins responded to antiprogestins. All clinical trials have been carried out in patients with advanced disease, who had frequently become resistant to other endocrine therapies.

\section{Conclusions and perspectives}

One treatment modality capable of curing breast cancer is still wishful thinking; however, the use of combined modalities is more realistic. The effects by which the progesterone/PR signaling pathway can be modulated are many, and the reviewed evidence from both experimental results and clinical trials clearly demonstrate that therapy. There is no doubt that antiprogestins in combination with other hormones/antihormones of proven antitumor effects such as tamoxifen, and inhibitors of ras, Src or MAPK will find their way into the routine treatment of breast cancer.

\section{Acknowledgements}

The authors are grateful to Consejo Nacional de Investigaciones Científicas y Técnicas, Fundación Sales and Laboratorios Gador for continuous support. They thank Dr I. Lüthy and Dr H. Coirini for discussion, and $\operatorname{Dr}$ C. D. Pasqualini for revising the manuscript.

\section{References}

1. Goepfert TM, McCarthy M, Kittrell FS, Stephens C, Ullrich RL, Brinkley BR, Medina D: Progesterone facilitates chromosome instability (aneuploidy) in p53 null normal mammary epithelial cells. FASEB J 2000, 14:2221-2229.

2. Women's Health Initiative: Risks and benefits of estrogen plus progestin in healthy postmenopausal women: principal results from the Women's Health Initiative randomized controlled trial. JAMA 2002, 288:321-333.

3. Ross RK, Paganini-Hill A, Wan PC, Pike MC: Effect of hormone replacement therapy on breast cancer risk: estrogen versus estrogen plus progestin. J Nat/ Cancer Inst 2000, 92:328-332.

4. Klijn JG, Setyono-Han B, Foekens JA: Progesterone antagonists and progesterone receptor modulators in the treatment of breast cancer. Steroids 2000, 65:825-830.

5. Lanari C, Molinolo AA, Pasqualini CD: Inhibitory effect of medroxyprogesterone acetate on foreign body tumorigenesis in mice. J Natl Cancer Inst 1986, 77:157-164.

6. Molinolo AA, Lanari C, Charreau EH, Sanjuan N, Pasqualini CD: Mouse mammary tumors induced by medroxyprogesterone acetate: immunohistochemistry and hormonal receptors. J Natl Cancer Inst 1987, 79:1341-1350.

7. Lanari C, Kordon E, Molinolo A, Pasqualini CD, Charreau EH: Mammary adenocarcinomas induced by medroxyprogesterone acetate: hormone dependence and EGF receptors of BALB/c in vivo sublines. Int J Cancer 1989, 43:845-850.

8. Nagasawa H, Aoki M, Sakagami N, Ishida M: Medroxyprogesterone acetate enhances spontaneous mammary tumorigenesis and uterine adenomyosis in mice. Breast Cancer Res Treat 1988, 12:59-66.

9. Kordon EC, Molinolo AA, Pasqualini CD, Charreau EH, Pazos P, Dran G, Lanari C: Progesterone induction of mammary carcinomas in BALB/c female mice. Correlation between progestin dependence and morphology. Breast Cancer Res Treat 1993, 28:29-39.

10. Pazos P, Lanari C, Meiss R, Charreau EH, Pasqualini CD: Mammary carcinogenesis induced by I-methyl-I-nitrosourea (MNU) and medroxyprogesterone acetate (MPA) in BALB/C mice. Breast Cancer Res Treat 1992, 20:133-138.

11. Aldaz CM, Liao QY, Paladugu A, Rehm S, Wang H: Allelotypic and cytogenetic characterization of chemically induced mouse mammary tumors: high frequency of chromosome 4 loss of heterozygosity at advanced stages of progression. Mol Carcinog 1996, 17:126-133.

12. Lydon JP, Ge G, Kittrell FS, Medina D, O'Malley BW: Murine mammary gland carcinogenesis is critically dependent on progesterone receptor function. Cancer Res 1999, 59:4276-4284.

13. Huggins C: Endocrine-induced regression of cancers. Science 1967, 156:1050-1054.

14. Lange CA, Richer JK, Horwitz KB: Hypothesis: progesterone primes breast cancer cells for cross-talk with proliferative or antiproliferative signals. Mol Endocrinol 1999, 13:829-836.

15. Lamb C, Simian M, Molinolo A, Pazos P, Lanari C: Regulation of cell growth of a progestin-dependent murine mammary carcinoma in vitro: progesterone receptor involvement in serum or growth factor-induced cell proliferation. J Steroid Biochem Mol Biol 1999, 70:133-142.

16. Richer JK, Jacobsen BM, Manning NG, Abel MG, Wolf DM, Horwitz KB: Differential gene regulation by the two progesterone receptor isoforms in human breast cancer cells. $J$ Biol Chem 2002, 277:5209-5218.

17. Shyamala G, Yang X, Cardiff RD, Dale E: Impact of progesterone receptor on cell-fate decisions during mammary gland development. Proc Natl Acad Sci USA 2000, 97:3044-3049. 
18. McKenna NJ, Lanz RB, O'Malley BW: Nuclear receptor coregulators: cellular and molecular biology. Endocr Rev 1999, 20: 321-344.

19. Giangrande $\mathrm{PH}, \mathrm{McD}$ onnell $\mathrm{DP}$ : The $\mathrm{A}$ and $\mathrm{B}$ isoforms of the human progesterone receptor: two functionally different transcription factors encoded by a single gene. Recent Prog Horm Res 1999, 54:291-313.

20. Clemm DL, Sherman L, Boonyaratanakornkit V, Schrader WT, Weigel NL, Edwards DP: Differential hormone-dependent phosphorylation of progesterone receptor $A$ and $B$ forms revealed by a phosphoserine site-specific monoclonal antibody. Mol Endocrinol 2000, 14:52-65.

21. Edwards DP, Weigel NL, Nordeen SK, Beck CA: Modulators of cellular protein phosphorylation alter the trans-activation function of human progesterone receptor and the biological activity of progesterone antagonists. Breast Cancer Res Treat 1993, 27:41-56

22. Jacobsen BM, Richer JK, Schittone SA, Horwitz KB: New human breast cancer cells to study progesterone receptor (PR) isoform ratio effects and ligand-independent gene regulation. J Biol Chem 2002, 277:27793-27800.

23. Elizalde $P$, Labriola L, Salatino M, Movsichoff F, Proietti C, Pecci A, Coso O, Kornblihtt A, Charreau E: Heregulin regulates the progesterone receptor $(\mathrm{Pr})$ transcriptional activity by a mechanism that requires a functional erbB-2 and mitogen-activated protein kinases (MAPK) activation. In Proceedings of the $A A C R$, New Orleans. Buenos Aires: Instituto de Biología y Medicina Experimental, and Facultad de Ciencias Exactas y Naturales, UBA; March 24-28, 2001.

24. Migliaccio A, Piccolo D, Castoria G, Di Domenico M, Bilancio A, Lombardi M, Gong W, Beato M, Auricchio F: Activation of the $\mathrm{Src} / \mathrm{p} 21$ ras/Erk pathway by progesterone receptor via crosstalk with estrogen receptor. EMBO J 1998, 17:2008-2018.

25. Maller JL: The elusive progesterone receptor in Xenopus oocytes. Proc Natl Acad Sci USA 2001, 98:8-10.

26. Bardon S, Vignon F, Montcourrier P, Rochefort H: Steroid receptor-mediated cytotoxicity of an antiestrogen and an antiprogestin in breast cancer cells. Cancer Res 1987, 47:1441-1448.

27. Vanzulli S, Efeyan A, Benavides F, Helguero L, Peters G, Shen J, Conti CJ, Lanari C, Molinolo A: p21, p27 and p53 in estrogen and antiprogestin-induced tumor regression of experimental mouse mammary ductal carcinomas. Carcinogenesis 2002, 23:749-757.

28. Nishino $Y$, Schneider MR, Michna H: Enhancement of the antitumor efficacy of the antiprogestin, onapristone, by combination with the antiestrogen, ICl 164384. J Cancer Res Clin Oncol 1994, 120:298-302. 